\title{
Early eradication therapy against Pseudomonas aeruginosa in cystic fibrosis patients
}

\author{
G. Taccetti*, S. Campana*, F. Festini*, M. Mascherini" ${ }^{*}$ and G. Döring"
}

ABSTRACT: In cystic fibrosis (CF) patients early antibiotic treatment of lung infection has been shown to lead to Pseudomonas aeruginosa eradication. The present study determined: 1) the time period from eradication to new $P$. aeruginosa acquisition; 2$) P$. aeruginosa re-growth and new acquisition; and 3 ) the impact of eradication therapy on lung function, antimicrobial resistance, emergence of other pathogens and treatment costs.

Ciprofloxacin and colistin were used to eradicate $P$. aeruginosa in $47 \mathrm{CF}$ patients. Bacterial pathogens, lung function decline, $P$. aeruginosa antimicrobial resistance and anti-pseudomonal serum antibodies were assessed quarterly and compared with an age-matched CF control group. Additionally, costs of antibiotic therapy in both groups were assessed.

Early antibiotic therapy leads to a $P$. aeruginosa free-period of a median (range) of 18 (4-80) months. New acquisition with different $P$. aeruginosa genotypes occurs in $73 \%$ of episodes. It also delays the decline of lung function compared with chronically infected patients, prevents the occurrence of antibiotic resistant $P$. aeruginosa strains, does not lead to emergence of other pathogens, and significantly reduces treatment costs. The treatment substantially lowers $P$. aeruginosa prevalence in CF.

In conclusion, early antibiotic therapy exerts beneficial effects on the patient's clinical status and is cost-effective compared with conventional antibiotic therapy for chronically infected cystic fibrosis patients.

\section{KEYWORDS: Antibiotic therapy, cystic fibrosis, Pseudomonas aeruginosa}

$\mathrm{n}$ the hereditary disease cystic fibrosis (CF), chronic bacterial lung infections are linked to mutations in a gene encoding the epithelial chloride channel, the CF transmembrane conductance regulator [1]. Decreased chloride secretion and increased sodium absorption leads to increased water absorption from the periciliary liquid layer on the respiratory epithelium, and the volume-depleted periciliary liquid layer impairs mucociliary clearance of bacterial organisms [1]. The opportunistic bacterial pathogen Pseudomonas aeruginosa is the most prevalent pathogen and accounts for most of the morbidity and mortality in CF patients. Although antibiotic therapy has considerably improved the clinical condition of CF patients and their quality of life since the 1980s [2], it rarely leads to eradication of the pathogen from chronically infected CF airways. P. aeruginosa macrocolony formation in anaerobic mucus plugs [3], and the occurrence of hypermutable $P$. aeruginosa strains, characterised by a rapid devel-

For editorial comments see page 373 . opment of antibiotic resistance, are thought to be responsible. Several studies have demonstrated that a delay of the onset of chronic $P$. aeruginosa infection and frequent eradication of the pathogen from CF airways is achieved when the antibiotic treatment is initiated shortly after bacterial lung colonisation [4-10]. This may result from findings that early after airway colonisation, single $P$. aeruginosa cells prevail, which are more susceptible to antibiotic attack. However, the early eradication treatment regimen has not yet been accepted worldwide and several open questions remain concerning: 1) the optimal drug (combination); 2) the mode of delivery of the antibiotic(s); 3) the optimal duration of the regimen; 4 ) the length of the $P$. aeruginosa free-period after treatment; and 5) the impact of this treatment strategy on lung function, development of antibiotic resistant microorganisms, emergence of other pathogens and treatment costs.

Thus, the objectives of the present study were to assess the time period after early antibiotic

\section{AFFILIATIONS}

${ }^{\star}$ Cystic Fibrosis Centre, Dept of Paediatrics, and

\#Dept of Statistics "G. Parenti",

University of Florence, Florence, Italy.

-Institute of Medical Microbiology and Hygiene, Universitätsklinikum Tübingen, Tübingen, Germany.

\section{CORRESPONDENCE}

G. Döring

Institute of Medical Microbiology and Hygiene

Universitätsklinikum Tübingen

Wilhelmstrasse 31

D-72074 Tübingen

Germany

Fax: 497071293011

E-mail: gerd.doering@med

uni-tuebingen.de

Received:

January 272005

Accepted after revision:

April 112005

SUPPORT STATEMENT

The financial support of TelethonItaly (grant no. GGP02205) and

support from the Fondazione per la Ricerca sulla Fibrosi Cistica (grant no. 18/2004) are gratefully

acknowledged. 
therapy when $P$. aeruginosa was again cultured from the patients' respiratory tract, and to differentiate between regrowth of an identical $P$. aeruginosa strain in a subsequent infection episode and re-infection with a different organism. Furthermore, the current authors wanted to assess the impact of early antibiotic therapy on the decline of lung function in comparison with that of antibiotic maintenance therapy in age-matched and sex-matched CF patients suffering from chronic $P$. aeruginosa infection. Additionally, the authors wanted to know whether antimicrobial resistance patterns of $P$. aeruginosa isolates from CF patients undergoing early eradication therapy differ from the resistance patterns of $P$. aeruginosa strains isolated from chronically infected $\mathrm{CF}$ patients. Furthermore, the present authors sought to know whether $P$. aeruginosa eradication would result in the emergence of other bacterial or fungal pathogens in CF airways. Finally, the costs of the early antibiotic therapy regimen were calculated and compared with those of antibiotic maintenance therapy in chronically infected CF patients over a 7-yr period.

\section{PATIENTS, MATERIALS AND METHODS}

At the CF centre in Florence (Italy) 173 CF patients, including $42 \%$ of patients aged $>18$ yrs, were seen quarterly. Cultures for $P$. aeruginosa and other CF-related pathogens from respiratory secretions were performed at every visit. During 1992-2001, all $P$. aeruginosa-negative CF patients who became $P$. aeruginosa positive as evidenced by one positive respiratory culture (29 males, 29 females; mean age \pm SD at time of first colonisation $8.9 \pm 6.64$ yrs) were immediately treated for 3 weeks with a combination of inhaled colistin $\left(1 \times 10^{-6}\right.$ international units (IU) b.i.d. $<6$ yrs; $2 \times 10^{-6} \mathrm{~mL}$ IU b.i.d. $>6 \mathrm{yrs}$ ) and oral ciprofloxacin $\left(30 \mathrm{mg} \cdot \mathrm{kg}^{-1} \cdot \mathrm{day}^{-1}\right)$. Treatment was prolonged to 3 months and all patients received $2 \times 10^{-6} \mathrm{~mL}$ IU b.i.d of colistin in cases when respiratory cultures did not become $P$. aeruginosa negative after the 3-week treatment [9].

Lung function, measured spirometrically (Renaissance PB100 apparatus; Nellcor Puritan Bennet, Pleasanton, CA, USA) as forced expiratory volume in one second (FEV1) related to ageadjusted reference values, was determined for a period of $5 \mathrm{yrs}$ in 18 patients submitted to $P$. aeruginosa eradication therapy and in 18 age-matched and sex-matched CF patients, chronically infected with $P$. aeruginosa. The CF patients of the control group had been chronically infected with $P$. aeruginosa in the period 1989-1992 before the treatment strategy was changed in the centre. Both patient groups did not differ statistically for CF genotype, pancreatic status, mean age, nutritional status, FEV1, patient and parent compliance, environmental exposure to pathogens, physician practices or socioeconomic status at the beginning of early eradication treatment. A patient was selected when monitoring of lung function was possible for $\geqslant 5$ yrs. Thus, particularly young patients who underwent early eradication therapy and in whom lung function data were not available were excluded. Patients were also excluded when a matched control individual was not available. The periods of lung function monitoring was comparable in the group of early treatment and the chronic infection group.

Antibiotics for treatment of pulmonary infections of $\mathrm{CF}$ patients are free in Italy according to law (law number 548, December 23, 1993). Antimicrobial susceptibility patterns of $P$. aeruginosa isolates from all patients were determined by the agar diffusion method. $P$. aeruginosa genotypes were assessed by pulsed field gel electrophoresis (PFGE) [11]. Briefly, bacterial DNA was cut with the restriction enzyme SpeI overnight at $37^{\circ} \mathrm{C}$ and electrophoresis was run at $200 \mathrm{~V}$ for $24 \mathrm{~h}$ with $1-25 \mathrm{~s}$ pulse times. Ethidium bromide stained DNA bands were scanned (Cybertech, Berlin, Germany), digitalised and analysed using the Wincam software program (Cybertech). There were no systematic differences between the individuals whose strains were genotyped and those where strains for genotyping were not available. Serum antipseudomonal antibodies were determined by ELISA [12]. Briefly, the purified $P$. aeruginosa antigens alkaline protease, elastase and exotoxin A, coated on albumin saturated microtiter plates were used to detect respective antibodies in $\mathrm{CF}$ serum samples. Additionally, serum samples were screened for anti-pseudomonal antibodies using crossed immunoelectrophoresis [13]. To assess whether early eradication therapy of $P$. aeruginosa leads to a change of the respiratory flora in $\mathrm{CF}$ patients, bacterial and fungal pathogens from respiratory cultures of $46 \mathrm{CF}$ patients were identified on agar plates before and after early eradication treatment. Between three to four cultures per patient, within periods of $8.2 \pm 3.4$ months before treatment and $8.8 \pm 5.9$ months after treatment, were assessed for: Staphylococcus aureus; methicillin-resistant S. aureus; Staphylococcus spp.; Burkholderia cepaci; Escherichia coli; Haemophilus influenzae; Klebsiella spp.; Stenotrophomonas maltophili; Acinetobacter spp.; Candida albicans; and Aspergillus fumigatus.

The cost of drugs used for early eradication treatment and for intravenous treatment of chronic $P$. aeruginosa lung infection was estimated for a 7-yr period [14].

\section{RESULTS}

Based on at least three consecutive negative respiratory $P$. aeruginosa cultures and negative serum antibody titers within a 6-month period after treatment, eradication of $P$. aeruginosa was achieved in $47 \mathrm{CF}$ patients $(81 \%)$ who had converted from $P$. aeruginosa negative to $P$. aeruginosa positive respiratory cultures. From these, 24 patients $(51 \%)$ were recolonised with the pathogen one or more times during the study period. Genotyping of $P$. aeruginosa strains, which were available from 50 isolated episodes of $P$. aeruginosa recolonisation in $16 \mathrm{CF}$ patients, revealed a different PFGE genotype in $73 \%$ of the subsequent episode (data not shown), further supporting the notion that eradication had been obtained in the majority of treated patients. There was no evidence for clonality of the $P$. aeruginosa $\mathrm{CF}$ isolates according to genotyping.

The mean \pm SD time period between two isolated episodes of $P$. aeruginosa colonisation in the early antibiotic treatment group of $47 \mathrm{CF}$ patients was a median (range) of 18 (4-80) months, suggesting that this treatment regimen has a beneficial impact on the patients' clinical status. Indeed, the average lung function decline (fig. 1) was significantly lower in early treated $\mathrm{CF}$ patients (mean $\pm \mathrm{SD} \Delta \mathrm{FEV} 1 \cdot \mathrm{yr}^{-1}:-1.63 \pm 1.60 \%$ ) than in chronically infected CF patients $\left(\Delta \mathrm{FEV} 1 \cdot \mathrm{yr}^{-1}\right.$ : $-4.69 \pm 2.95 \%$; unpaired t-test: $\mathrm{p}<0.05)$. Lung function could not be assessed in all of the patients in the early antibiotic treatment group due to ages $<5$ yrs.

$P$. aeruginosa isolates from early treated patients versus control patients were significantly more sensitive to the 


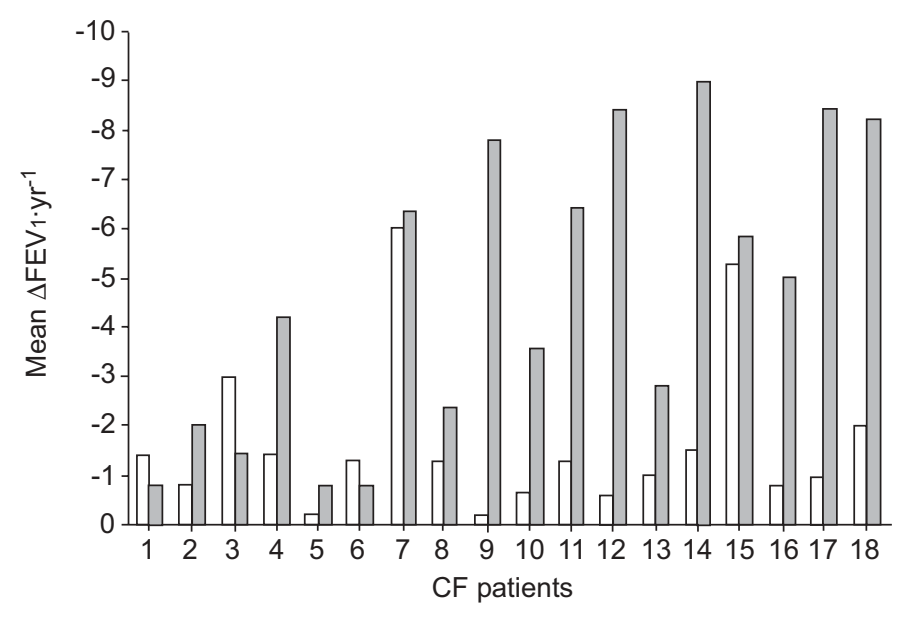

FIGURE 1. The decrease of lung function (average forced expiratory volume in one second $\left.(\Delta \mathrm{FEV} 1) \cdot \mathrm{yr}^{-1}\right)$ determined in a 5 -yr period in 18 cystic fibrosis (CF) patients treated early with inhaled colistin/oral ciprofloxacin $(\square)$ and in 18 agematched CF patients chronically infected with Psuedomonas aeruginosa ( $\square$ ).

$P$. aeruginosa-specific antibiotics (Chi-squared test: $p<0.05$ ) ceftazidime, tobramycin and ciprofloxacin and revealed a nonmucoid phenotype (fig. 2). The data show that $P$. aeruginosa is much more susceptible to antibiotics when therapy is administered early after lung colonisation.

Since eradication of $P$. aeruginosa may result in the emergence of other pathogens in the respiratory tract, bacterial and fungal pathogens from CF patients were identified before and after early eradication treatment (table 1). No statistical difference was obtained for the investigated bacterial or fungal pathogens.

The 47 early-treated patients received a total of 104 antibiotic "maintainance" therapy courses over 7 yrs (0.32 courses. patient $\left.\cdot \mathrm{yr}^{-1}\right)$ costing in total $€ 34,681\left(€ 105.4 \cdot\right.$ patient $\left.\cdot \mathrm{yr}^{-1}\right)$. The 47 age-matched, chronically $P$. aeruginosa-infected CF patients received 683 courses in 7 yrs $\left(2.1\right.$ courses $\cdot$ patient $\left.\cdot \mathrm{yr}^{-1}\right)$ costing in total $€ 1,767,025\left(€ 5371 \cdot\right.$ patient $\left.\cdot \mathrm{yr}^{-1}\right)$.

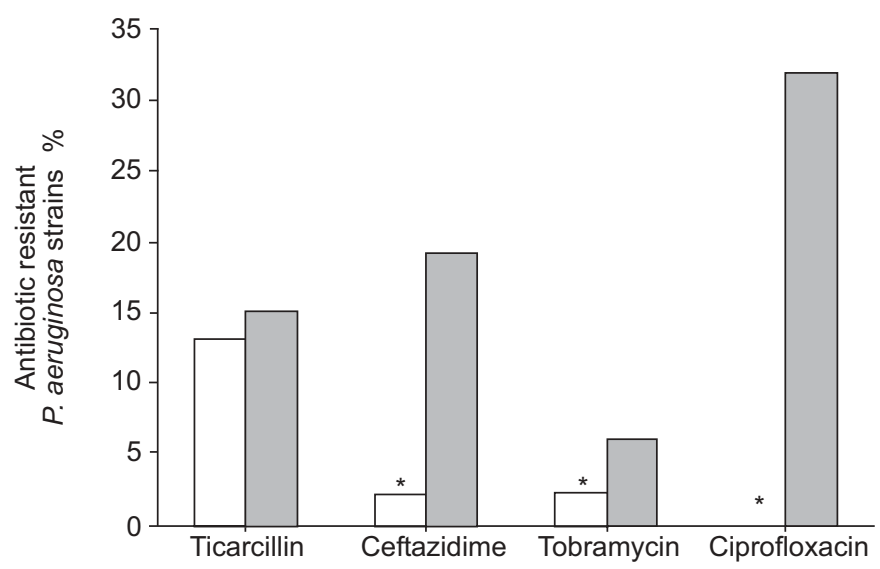

FIGURE 2. Antibiotic resistance patterns of 47 Pseudomonas aeruginosa isolates from 47 early treated patients $(\square)$ and 47 isolates from 47 chronically infected patients $(\square)$. *: $p<0.05$.

\begin{tabular}{|c|c|c|c|}
\hline TABLE 1 & \multicolumn{3}{|c|}{$\begin{array}{l}\text { Frequency of bacterial and fungal pathogens } \\
\text { before and after eradication therapy in specimens } \\
\text { of CF patients }\end{array}$} \\
\hline \multirow{2}{*}{\multicolumn{2}{|c|}{ Pathogen }} & $\begin{array}{c}\text { Before } \\
\text { eradication }\end{array}$ & $\begin{array}{c}\text { After } \\
\text { eradication }\end{array}$ \\
\hline & & \multicolumn{2}{|c|}{ Positive cultures } \\
\hline \multicolumn{2}{|c|}{ Staphylococcus aureus } & $133(648)$ & $183(78.5)$ \\
\hline \multicolumn{2}{|l|}{ MRSA } & 0 & $2(0.8)$ \\
\hline \multicolumn{2}{|c|}{ Coagulase-negative staphylococcus } & $25(12)$ & $10(4.2)$ \\
\hline \multicolumn{2}{|c|}{ Burkholderia cepacia complex } & 0 & $1(0.4)$ \\
\hline \multicolumn{2}{|c|}{ Escherichia coli } & $19(9.2)$ & $6(2.5)$ \\
\hline \multicolumn{2}{|c|}{ Haemophilus influenzae } & $19(9.2)$ & $11(4.7)$ \\
\hline \multicolumn{2}{|c|}{ Klebsiella spp. } & $7(3.4)$ & $8(3.4)$ \\
\hline \multicolumn{2}{|c|}{ Stenotrophomonas maltophilia } & $4(1.9)$ & $8(3.4)$ \\
\hline \multicolumn{2}{|c|}{ Achromobacter xylosoxidans } & $1(0.4)$ & $2(0.8)$ \\
\hline \multicolumn{2}{|c|}{ Acinetobacter spp. } & $2(0.9)$ & $1(0.4)$ \\
\hline \multicolumn{2}{|c|}{ Candida albicans } & $43(20.9)$ & $25(10.7)$ \\
\hline \multicolumn{2}{|c|}{ Aspergillus fumigatus } & $10(4.8)$ & $18(7.7)$ \\
\hline \multicolumn{2}{|l|}{ Normal flora } & $18(8.7)$ & $18(7.7)$ \\
\hline
\end{tabular}

\section{DISCUSSION}

The present study answers several important questions. The period during which the patients remained free of $P$. aeruginosa after a 3 week to 3 month eradication therapy is encouragingly lengthy. In previous studies, the period in which the patients remained free of $P$. aeruginosa after early therapy was determined to be 3-25 months [6], and in one study $>1 \mathrm{yr}$ [7]. Apparently, eradication is not immediately followed by reinfection of the patients with $P$. aeruginosa strains ubiquitously present in the environment. The treatment regimen also lead to true eradication as demonstrated by the diversity of genotyped sequential $P$. aeruginosa isolates from the majority of $\mathrm{CF}$ patients and by negative specific antibody titers against $P$. aeruginosa.

The Damocles sword of antibiotic resistance and adverse side effects is a common threat to conventional antibiotic therapy for chronically P. aeruginosa-infected CF patients, which has led to restrictive antibiotic treatment strategies in various $\mathrm{CF}$ centres. Early antibiotic therapy circumvents this problem efficiently. Since $P$. aeruginosa strains infecting CF airways are highly sensitive to many $P$. aeruginosa specific antibiotics, $P$. aeruginosa eradication is often achieved and the development of antibiotic resistance becomes marginal. The high success rate makes it conceivable that early antibiotic treatment has a beneficial impact on lung function compared with chronically infected CF patients and, thus, may well result in a higher life expectancy of early treated CF patients.

A further positive aspect of early antibiotic treatment of $P$. aeruginosa in CF concerns the costs of treatment. Compared with regular intravenous antibiotic therapy for chronically infected CF patients [2], the cost for early antibiotic treatment is $\sim 2 \%$, since $P$. aeruginosa eradication leaves the patient for prolonged periods of time free of the pathogen. Besides the 
positive clinical aspects, the significantly reduced costs will undoubtedly make this therapy regimen attractive in many countries in which CF patients live. Reduced costs of early antibiotic treatment were also calculated by others [15]. If widely applied it may substantially reduce the prevalence of chronic $P$. aeruginosa lung infections in CF patients. At present, only $39.3 \%$ of CF patients in the Cystic Fibrosis Centre of Tuscany (Meyer Pediatric Hospital, University of Florence, Italy) suffer from chronic $P$. aeruginosa lung infections as a result of early antibiotic therapy. Decreases of the prevalence of chronic $P$. aeruginosa lung infections in CF patients have also been reported from other centres [16]. A larger cohort of patients needs to be studied to see if this development will lead to an increase of the prevalence of other pathogens in the respiratory tract. No increase in a number of CF-related bacterial and fungal pathogens were noticed after early treatment of $P$. aeruginosa, however, prolonged studies are needed to confirm this finding. An opposite development was observed in a group of young CF patients undergoing prophylactic treatment against $S$. aureus. The decrease of $S$. aureus was correlated with a significant increase in the number of patients harbouring P. aeruginosa.

In conclusion, taken together, the results of this study show that early antibiotic therapy for eradication of Pseusomonas aeruginosa is a powerful therapy regimen, which substantially reduces the prevalence of chronic Pseudomonas aeruginosa lung infections in cystic fibrosis patients.

\section{REFERENCES}

1 Ratjen F, Döring G. Cystic fibrosis. Lancet 2003; 361: 681-689.

2 Döring G, Conway SP, Heijerman HGM, et al, for the Consensus Committee. Antibiotic therapy against Pseudomonas aeruginosa in cystic fibrosis: a European consensus. Eur Respir J 2000; 16: 749-767.

3 Worlitzsch D, Tarran R, Ulrich M, et al. Reduced oxygen concentrations in airway mucus contribute to the early and late pathogenesis of Pseudomonas aeruginosa cystic fibrosis airways infection. J Clin Invest 2002; 109: 317-325.

4 Valerius NH, Koch C, Høiby N. Prevention of chronic Pseudomonas aeruginosa colonisation by early treatment. Lancet 1991; 338: 725-726.

5 Wiesemann HG, Steinkamp G, Ratjen F, et al. Placebocontrolled, double-blind, randomized study of aerosolized tobramycin for early treatment of Pseudomonas aeruginosa colonization in cystic fibrosis. Pediatr Pulmonol 1998; 25: 88-92.

6 Munck A, Bonacorsi S, Mariani-Kurkdjian P, et al. Genotypic characterization of Pseudomonas aeruginosa strains recovered from patients with cystic fibrosis after initial and subsequent colonization. Pediatr Pulmonol 2001; 32: 288-292.

7 Ratjen F, Döring G, Nikolaizik WH. Effect of inhaled tobramycin on early Pseudomonas aeruginosa colonisation in patients with cystic fibrosis. Lancet 2001; 358: 983-984.

8 Gibson RL, Emerson J, McNamara S, et al. Significant microbiological effect of inhaled tobramycin in young children with cystic fibrosis. Am J Respir Crit Care Med 2003; 167: 841-849.

9 Taccetti G, Repetto T, Procopio E, Farina S, Campana S. Early Pseudomonas aeruginosa colonization in cystic fibrosis patients. Lancet 2002; 359: 625-626.

10 Littlewood JM, Miller MG, Ghoneim AT, Ramsden $\mathrm{CH}$. Nebulised colomycin for early Pseudomonas aeruginosa colonisation in cystic fibrosis. Lancet 1985; i: 865.

11 Döring G, Jansen S, Noll $\mathrm{H}$, et al. Distribution and transmission of Pseudomonas aeruginosa and Burkholderia cepacia in a hospital ward. Pediatr Pulmonol 1996; 21: 90-100.

12 Danielsen L, Westh H, Balselv E, Rosdahl VT, Döring G. Pseudomonas aeruginosa exotoxin A antibodies in rapidly deteriorating chronic leg ulcers. Lancet 1996; 347: 265.

13 Høiby N, Anderson V, Bendixen G. Pseudomonas aeruginosa infection in cystic fibrosis. Humoral and cellular immune responses against Pseudomonas aeruginosa. Acta Pathol Microbiol Scand (C) 1975; 83: 459-468.

14 Martini N, Addis A, Amato P, et al. Il nuovo prontuario farmaceutico nazionale. [Italian Directory of Medicine]. 63rd Edn. Milan, Masson, 2003.

15 Baumann U, Stocklassa C, Greiner W, Graf von der Schulenburg J-M, von der Hardt H. Costs of care and clinical conditions in paediatric cystic fibrosis patients. $J$ Cyst Fibros 2003; 2: 84-90.

16 Frederiksen B, Koch C, Hoiby N. Antibiotic treatment of initial colonization with Pseudomonas aeruginosa postpones chronic infection and prevents deterioration of pulmonary function in cystic fibrosis. Pediatr Pulmonol 1997; 23: 330-335. 